Dyulmesova-Bilash Olga. Clinical considerations for the management of psoriasis in women with disorders of the menstrual cycle. $\begin{array}{llllll}\text { Journal of Education, Health and Sport. 2021;11(09):816-827. } & \text { eISSN 2391-8306. }\end{array}$ http://dx.doi.org/10.12775/JEHS.2021.11.09.097

https://apcz.umk.pl/JEHS/article/view/JEHS.2021.11.09.097

https://zenodo.org/record/5585626

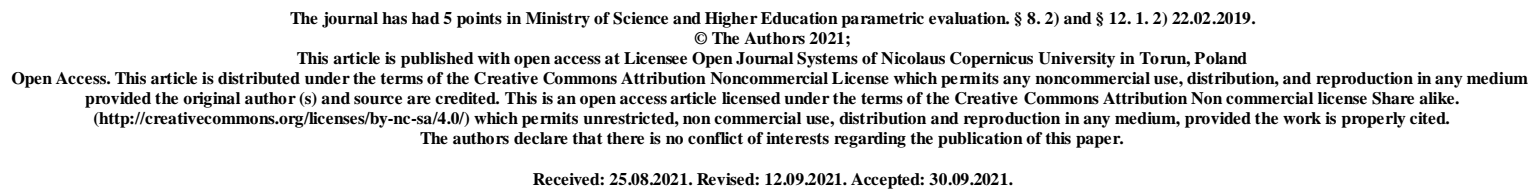

UDC: $616.517 .8+618.11-008.64: 616-035.1$

\title{
CLINICAL CONSIDERATIONS FOR THE MANAGEMENT OF PSORIASIS IN WOMEN WITH DISORDERS OF THE MENSTRUAL CYCLE
}

\author{
Olga Dyulmesova-Bilash
}

\author{
Zaporozhye State Medical University, \\ Maiakovskyi avenue 26, Zaporizhzhia, Ukraine, 69035 \\ Department of Dermatovenereology and cosmetology with a course in dermatovenereology \\ and Aesthetic Medicine, Faculty of Postgraduate Education, \\ Full-time graduate student
}

\begin{abstract}
Introduction. Psoriasis in women of reproductive age is one of the most pressing medical and social problems. The consequence of psoriasis disease is a significant decrease in the quality of life, disability of patients, difficulties in creating a family and deterioration of family relations, significant psychological discomfort.

Objective of the study: to increase the effectiveness of treatment of women with psoriasis with menstrual irregularities by means of pathogenetically substantiated therapy based on the study of hormonal changes.

Materials and research methods. The study included 130 women who were divided into three groups: the main group - 75 women with psoriasis with menstrual irregularities, who were randomized into 3 subgroups: $1 \mathrm{a}-25$ patients who will receive standard psoriasis therapy; $1 \mathrm{~b}-25$ patients, in addition to the standard treatment of psoriasis, will receive
\end{abstract}


gestagens for 3 months; $1 \mathrm{c}-25$ patients, in addition to the standard treatment of psoriasis, will receive a preparation of an dry extract of herb of creeping anchors for 3 months. The comparison group consisted of 25 women with psoriasis without hormonal disorders, comparable in age and social status with the study group. The control group - 30 apparently healthy women, comparable in age and social status with the study group.

Research results. In patients of the main group who received standard psoriasis therapy (1a); who received progestogens for 3 months in addition to standard psoriasis treatment (1b) and, in addition to the standard treatment of psoriasis, who received the preparation of an dry extract of herb of anchors creeping for 3 months (1c), before the start of the treatment, the ratio of $\mathrm{LH} / \mathrm{FSH}$ - luteinizing hormone ( $\mathrm{LH})$ and follicle-stimulating hormone (FSH) was below normal, which indicates a certain deficit in LH production relative to FSH values. In parallel, in these cohorts, the production of prolactin was increased, which suppresses the formation of FSH and LH in the pituitary gland. Changes in the production of gonadotropic hormones are a reaction to the existing deficiency or excess of sex hormones. In the main 1a, $1 \mathrm{~b}$ and $1 \mathrm{c}$ in groups, estradiol values were within the normal range with progesterone deficiency in the luteal phase. The testosterone value in all study groups did not deviate from the standard values.

Conclusions. The obtained results of the survey indicate the need to increase the effectiveness of treatment of women with psoriasis with menstrual irregularities by involving measures aimed at correcting hormonal status in complex therapy.

Keywords: psoriasis; follicle-stimulating hormone; luteinizing hormone; prolactin; testosterone; progesterone; estradiol.

Revalence. Psoriasis in women of reproductive age is one of the most pressing medical and social problems. The consequence of psoriasis disease is a significant decrease in the quality of life, disability of patients, difficulties in creating a family and deterioration of family relations, significant psychological discomfort [1].

In recent years in Ukraine has seen an increase in the incidence of psoriasis. The number of patients with severe disabling forms of psoriasis, resistant to various therapies, is also increasing [2].

This is due to many factors, including urbanization and the negative impact of the environment, especially in large industrial cities, environmental or social ills (chronic stress), widespread human use of chemicals, xenobiotics, and malnutrition [3]. 
There are numerous theories about the occurrence of psoriasis, but none of them can be the only cause. The main theories of the origin and development of psoriasis include: genetic, neurogenic, infectious, parasitic, viral, endocrine-metabolic and others. However, many other trigger factors play a role in the onset and progression of psoriasis, most of which require appropriate research to further develop measures to control psoriasis [4].

Of particular note is the prevalence of psoriasis in the female population, which is due to the peculiarities of hormonal status, which varies with age and phases of the menstrual cycle. The presence of concomitant changes in the levels and ratio of sex hormones leads to an increase in the percentage of patients with frequent relapses and the presence of resistance to various treatments [5].

Despite the large number of publications to date, not all factors of the etiology and pathogenesis of psoriasis have been definitively elucidated, especially in women of reproductive age depending on hormonal status [6].

Aim of the study. To increase the effectiveness of treatment of women with psoriasis with menstrual irregularities by means of pathogenetically substantiated therapy based on the study of hormonal changes.

Materials and methods of research. The study included 130 women, who were divided into three groups:

1. The main group - 75 patients with psoriasis with menstrual disorders, which by randomization were divided into 3 subgroups: 1a - 25 patients who will receive standard therapy for psoriasis; $1 \mathrm{~b}-25$ patients who, in addition to the standard treatment of psoriasis, will receive progestogens for 3 months; 1c - 25 patients who, in addition to the standard treatment of psoriasis, will receive a preparation of dry extract of herb of creeping anchors for 3 months.

2. Comparison group - 25 patients with psoriasis without hormonal disorders, comparable in age and social status with the study group.

3. Control group - 30 healthy women, comparable in age and social status with the study group.

Patients from the main group were examined before treatment, 3 and 6 months after its start.

The criteria for inclusion in the study were as follows: signed informed consent to participate in the study; women aged 18 to 45; patients with psoriasis.

Exclusion criteria: existing uncompensated liver disease or past liver disease if liver function tests have not returned to normal; the presence of other severe somatic pathology; the 
presence of other dermatological pathology that may affect the results of the study; established or suspected progestogen-dependent neoplasms (eg, meningioma).

The study was conducted in accordance with the basic bioethical norms of the "Declaration of Helsinki. World Medical Association Declaration of Helsinki. Ethical Principles for Medical Research Involving Human Subjects" (2000, as amended in 2008), the Universal Declaration on Bioethics and Human Rights (1997), the Convention on Human Rights and Biomedicine developed by the Council of Europe (1997). Written informed consent was obtained from each study participant.

Clinical and anamnestic (diagnosis, diagnosis of exacerbation of dermatosis, evaluation of the effectiveness of treatment of previous therapies) and general clinical methods (complete blood test, complete urine examination, blood glucose test, alanine aminotransferase, aspartate transaminase, total bilirubin, creatinine, detection of helminth eggs in stool samples, serological tests for syphilis). The social status and quality of life of patients in each group were also assessed as part of a sociological study.

Enzyme-linked immunosorbent assay was used to determine the levels of folliclestimulating hormone $(\mathrm{FSH})$, luteinizing hormone $(\mathrm{LH})$, prolactin, testosterone, progesterone and estradiol in the serum. Enzyme-linked immunosorbent assay DRG HPL № BS-85-24 (Germany) is a solid-phase, enzyme-labeled, immunosorbent assay (ELISA) based on the "sandwich" principle. Microplate wells are coated with monoclonal antibodies directed against a unique antigenic site on the molecule of the above hormones. An aliquot of a sample of a patient with endogenous hormone is incubated in a well with an enzyme conjugate, which is a monoclonal anti-hormone antibody conjugated to horseradish peroxidase. After incubation, the unbound conjugate is washed away. The amount of bound peroxidase is proportional to the concentration of the corresponding hormone in the sample. When adding the substrate solution, a color appears, the intensity of which is proportional to the concentration of hormones in the patient's sample.

The distribution normality analysis was evaluated according to the preferred ShapiroWilk (W) criteria. When it was impossible to reject the null hypothesis of statistically significant differences in the distribution of variables from normal, nonparametric data analysis methods were used, and in other cases, parametric methods. Data are presented as mean and standard error of representativeness of the sample mean (in the case of normal distribution), and in the form of median and interquartile range (25-75 percentile, if there is a difference from the normal distribution). In the case of a distribution other than normal or analysis of ordinal variables, the Mann-Whitney U-test was used for 2 unbound samples, and 
for a larger number of samples, the Kruskal-Wallis $\mathrm{H}$ test was followed by a Games-Howell comparison. Comparison of the two groups was performed using the Wilcoxon test.

Research results. In many works devoted to the endocrine component of psoriasis, changes in certain hormones are considered as a basis against which pathological processes in the skin unfold. In this case, the disruption of the endocrine effectors is interpreted both as an inducer of hyperproliferative skin processes, and as their accelerator.

There is a lot of data about the role of stress in the development of psoriasis, and on the disruption of the hypothalamic-pituitary-adrenal axis in this disease. It has been shown that cortisol is capable of activating the proliferation of keratinocytes in vitro. Along with the effect of glucocorticosteroids on keratinocytes, these hormones in high concentrations are capable of causing a pronounced immunosuppressive effect. The level of cortisol in patients with psoriasis in the progressive stage in one of the studies was approximately doubled compared with the norm, but decreased in the stationary phase. In other studies, patients with psoriasis have decreased cortisol levels. During the progression of the disease, there is a urinary secretion of glucocorticoids and promineralocorticoids. At the same time, iatrogenic inhibition of cortisol causes a sharp exacerbation of psoriasis.

There were determined the dynamics of changes in sex hormones in women of the study groups in the luteal phase before therapy. In patients of the main group, who received standard therapy for psoriasis (1a), the level of FSH and LH was 7.13 \pm 7.21 and $9.56 \pm 1.24$ $\mathrm{ng} / \mathrm{ml}(\mathrm{p}<0.05)$, respectively, and the ratio of these hormones LH / FSH, which has a normative value of 1.5-2.0, was 1.34, which indicates a certain deficit of LH production relative to FSH values. In parallel, increased prolactin production to $26.14 \pm 2.74 \mathrm{ng} / \mathrm{ml}$, which inhibits the formation of FSH and LH in the pituitary gland. Changes in the production of gonadotropic hormones are a reaction to an existing deficiency or excess of sex hormones. In the main 1a group, estradiol values are $61.15 \pm 5.46 \mathrm{pg} / \mathrm{ml}(\mathrm{p}<0.05)$ with progesterone deficiency in the luteal phase $-7.13 \pm 0.68 \mathrm{ng} / \mathrm{ml}(\mathrm{p}<0.05)$. Testosterone values in the main 1a group were found at $2.38 \pm 1.75 \mathrm{nmol} / 1$.

For women in the main group, who received progestogens for 3 months in addition to standard psoriasis treatment (1b), the following levels of gonadotropic hormones were detected: FSH $6.76 \pm 5.47 \mathrm{ng} / \mathrm{ml}$ and LH $10.22 \pm 1.19 \mathrm{ng} / \mathrm{ml}(\mathrm{p}<0,05)$, the ratio of LH / FSH is 1.51. Prolactin levels were slightly lower than in the previous group $-19.32 \pm 2.26 \mathrm{ng}$ $/ \mathrm{ml}$. There is also a decrease in progesterone levels in group $1 \mathrm{~b}-8.28 \pm 0.74 \mathrm{ng} / \mathrm{ml}(\mathrm{p}$ $<0.05$ ) in the luteal phase with sufficient levels of estradiol for this phase $-78.23 \pm 9.14 \mathrm{pg} /$ 
$\mathrm{ml}(\mathrm{p}<0.05)$. For the main $1 \mathrm{~b}$ group, testosterone was determined within normal limits -2.87 $\pm 2.15 \mathrm{nmol} / \mathrm{l}(\mathrm{p}<0.05)$.

In patients of the main group, who in addition to the standard treatment of psoriasis received a preparation of dry extract of herb of creeping anchors for 3 months (1c), the level of FSH and LH was $8.29 \pm 7.15$ and $11.42 \pm 1.31 \mathrm{ng} / \mathrm{ml}(\mathrm{p}<0.05)$, respectively, and the ratio of these LH / FSH hormones was 1.38, which also indicates a deficit of LH production relative to FSH values. Increased prolactin production to $28.51 \pm 3.37 \mathrm{ng} / \mathrm{ml}$. In the main $1 \mathrm{c}$ group, estradiol values are $71.84 \pm 7.76 \mathrm{pg} / \mathrm{ml}(\mathrm{p}<0.05)$ with progesterone deficiency in the luteal phase $-5.96 \pm 0.61 \mathrm{ng} / \mathrm{ml}(\mathrm{p}<0.05)$. Testosterone values in the main 1c group were found at $3.12 \pm 2.81 \mathrm{nmol} / 1$.

The following levels of gonadotropic hormones were found for women in the comparison group with psoriasis without dyshormonal disorders: FSH $7.67 \pm 6.81 \mathrm{ng} / \mathrm{ml}(\mathrm{p}$ $<0.05$ ) and $\mathrm{LH} 13.09 \pm 1.57 \mathrm{ng} / \mathrm{ml} \mathrm{p}<0,05)$, the ratio of LH / FSH is 1.71. Prolactin levels were slightly lower than in previous cohorts of the main group $-17.41 \pm 1.65 \mathrm{ng} / \mathrm{ml}$. Progesterone levels in the comparison group were within normal limits $(15.35 \pm 1.12 \mathrm{ng} / \mathrm{ml})$ in the luteal phase at sufficient levels of estradiol for this phase $-191.81 \pm 17.48 \mathrm{pg} / \mathrm{ml}(\mathrm{p}$ $<0.05)$. For this comparison group, testosterone was also determined within normal limits $1.46 \pm 1.09 \mathrm{nmol} / \mathrm{l}(\mathrm{p}<0.05)$.

In the control group of 30 almost healthy women there were no deviations in the values of sex hormones in the luteal phase of the cycle: FSH $-9.13 \pm 1.07 \mathrm{ng} / \mathrm{ml}, \mathrm{LH}-$ $17.89 \pm 2.14 \mathrm{ng} / \mathrm{ml}$, the ratio of LH / FSH -1.96 , prolactin $-16.62 \pm 0.89 \mathrm{ng} / \mathrm{ml}$, estradiol $-214.81 \pm 19.35 \mathrm{pg} / \mathrm{ml}$, progesterone $-17.42 \pm 0.93 \mathrm{ng} / \mathrm{ml}$, testosterone $-1.23 \pm 1.17$ nmol/ 1 .

The development of the disease is accompanied by a high level of prolactin in the blood of patients with psoriasis. However, the induced secretion in the pituitary gland is reduced, which indicates a decrease in the reserves of prolactin in the gland. The role of prolactin in the pathogenesis of psoriasis is confirmed by the fact that pregnancy and lactation are one of the psoriasis-provoking factors. At the same time, according to the data of other authors, the content of prolactin in psoriasis may not increase, or even decrease in comparison with the norm. Thus, the role of prolactin in the pathogenesis of psoriasis remains unclear to date.

The content of testosterone in the plasma of patients with psoriasis can be both normal and low; a decrease in DHEA (dehydroepiandrosteron) and DHEA sulfate is also described, which can be considered as a marker of suppression of adrenal androgenic function. A 
decrease in the concentration of testosterone can reduce the intensity of anabolic processes in the body of patients with psoriasis, and lead to an increase in the concentration of glucose in the blood. In addition, testosterone can directly influence keratinocyte proliferation through increased fatty acid synthesis. Thus, carrying out a systematic study of hormonal changes in psoriasis in women and comparing them with a complex of clinical manifestations is necessary.

To characterize the pathogenetic significance of changes in the secretion of gonadotropic and sex hormones in the development of psoriasis in women, ROC analysis was used to determine the values of differential diagnostic cut-off points and the corresponding parameters of diagnostic sensitivity and specificity in women according to the prognosis of the development of acute forms of psoriasis. For this, a statistical matrix was compiled, where the presence or absence of acute forms of psoriasis in women in the main, comparison and control groups was marked 1 or 0 and the corresponding values of hormones were presented.

In women the risk of developing of acute forms of psoriasis increased with an increase in the blood FSH content above $48 \mathrm{mMe} / \mathrm{ml}$ (sensitivity $-87 \%$, specificity - 84\%), LH - above $27 \mathrm{mMe} / \mathrm{ml}$ (sensitivity $-81 \%$, specificity $-82 \%$ ), decrease in prolactin - below $348 \mathrm{mMe} / \mathrm{ml}$ (sensitivity - 94\%, specificity - 70\%), estradiol - below $21 \mathrm{pg} / \mathrm{ml}$ (sensitivity $-93 \%$, specificity $-77 \%$ ), an increase in testosterone above $0.47 \mathrm{ng} / \mathrm{ml}$ (sensitivity $-89 \%$, specificity $-60 \%$ ). Changes in the LH / FSH index, the level of progesterone in the blood in women are not associated with the risk of development of acute forms of psoriasis. Consequently, one of the triggering stimuli for the development of acute forms of psoriasis in women was hypergonadotropic ovarian failure.

Discussion. Psoriasis affects women of all ages, including reproductive [7]. Estrogen and progesterone levels rise steadily throughout pregnancy until the prenatal period. The team reported patients taking high-dose oral estrogen contraceptives and showing an overall improvement in psoriasis, while a study of 92 patients concluded that there was little evidence of any effect of oral contraceptives on psoriasis. Some studies have found a different correlation in the observation of a patient in whom psoriasis responded positively to the administration of the antiestrogen drug tamoxifen [8].

During a woman's reproductive age, stressful life events, such as pregnancy, which are characterized by multiple physiological changes, affect the development of psoriasis and affect its clinical manifestation. Sex hormones, especially estrogen and prolactin, play an important role in modulating the immune response. Prolactin, secreted by the pituitary gland, 
as well as other organs and cells, has an immunostimulatory effect and promotes autoimmunity. It inhibits the induction of B-cell tolerance, enhances the proliferative response to antigens and mitogens, increases the production of immune globulins, cytokines and autoantibodies. Patients with hyperprolactinemia have many different clinical manifestations, one of which is psoriasis. There are data indicating a correlation between prolactin levels and disease activity [9]. The link between neurotransmitters, hormones and other stress-related factors and exacerbation of certain immunopathological conditions, such as psoriasis, has been documented. Increased production of vascular endothelial growth factor (VEGF) in macrophages is stimulated by estrogen, an effect that is antagonized by androgens, and it is believed that the imbalance in the ratio of hormones may be associated with the development of dermatological diseases during pregnancy. The potential role of sex hormones in the etiology of psoriasis has been proven, as pregnancy, a state of natural immunomodulation, is associated with the relief or exacerbation of various inflammatory diseases, including psoriasis [10].

Psoriasis in children and adolescents is not uncommon, and many studies indicate the appearance of psoriatic lesions up to 16 years in one third of patients. It was noted that $63.5 \%$ of patients are ill before the age of 30 [11]. The age of onset of the disease was documented, according to the literature, approximately during puberty, as well as between 30 and 50 years. This bimodal age was also initially described by several authors who report middle-aged people with onset of psoriasis in the range of 15 to 20 years in 2147 patients, with a second peak at the age of 55-60 years. There is a link between psoriasis and hormonal changes at different stages of life; however, it has not yet been reliably identified [12]. In women, hormonal changes similar to those that occur during puberty can cause or exacerbate psoriasis, as mentioned in some studies that have looked at the relationship between the development of psoriasis and puberty and found that in many people the first psoriatic lesions develop immediately after puberty, which correlates with a decrease in hormone levels. A recent study found that sex hormones have different biological and immune effects in the skin, and fluctuations in hormone levels during a woman's menstrual cycle can affect psoriasis. During the menstrual cycle, the follicle in the ovary actively secretes estrogen until their level in the serum reaches a threshold value. After about 10 days, the corpus luteum begins to degenerate, with estrogen and progesterone levels decreasing on approximately day 26 of the cycle. LH levels begin to rise, and therefore the follicles are stimulated to mature, so that by the beginning of a new cycle, estrogen levels rise again [13]. It is reported that menstruation is associated with modulation of the natural course of psoriasis, which suggests 
that inflammation of the skin may be caused by hormonal changes. It was noted that estrogen inhibits the production of neutrophils, $\mathrm{T}$ cells and chemokines that stimulate macrophages, CXCL8, CXCL10 and CCL5, inhibits the production of IL-12 and antigen-presenting ability of cells, while enhancing the anti-inflammatory IL-10 production of dendrites, indicating how inflammation in psoriatic lesions may be related to estrogen [14].

Elevated levels of sex hormones, particularly estrogen, which are known to promote keratinocyte proliferation through a special receptor-mediated mechanism, may explain the increase in the prevalence of psoriasis in the period before menarche. This mechanism, apparently, plays an important role in the process of wound healing, it is also assumed that this effect alone can provide a significant stimulus to the development of epidermal hypertrophy characteristic of psoriasis [15].

It is also known that sex hormones affect the appearance and course of inflammation. Elevated estrogen levels during menarche can affect Th1 and Th2 immune responses through cytokines and chemokines, including the production of monocyte chemoattractant protein-1 (MCP-1). These changes can stimulate both cellular activity and the inflammatory response caused by tumor necrosis factor TNF-alpha, potentially providing a more direct link with the pathophysiology of psoriasis [16].

In general, it can be assumed that high estrogen levels appear to have a more regulatory and inhibitory effect on many components of the immune response, while low levels can be stimulating [17]. These various regulatory effects of sex steroids and their fluctuations during puberty and adolescence have been associated with many skin conditions, including psoriasis, and are the focus of many therapeutic or prophylactic measures. It is important for the patient and the physician to understand that psoriasis is a chronic disease and that hormonal changes can affect the course of the disease. Patients need to understand that psoriasis can recur throughout life, and that it is important to understand the relationship between the disease and the hormonal phases that can worsen it [18].

Limitations in research. Financial resources, method and data collection.

Prospects for further research. Prospects for further research are to study the features of effectiveness of treatment of women with psoriasis with menstrual irregularities by means of pathogenetically substantiated therapy based on the study of clinical changes.

Conclusions. In patients of the main group who received standard psoriasis therapy (1a); who received progestogens for 3 months in addition to standard psoriasis treatment (1b) and, in addition to the standard treatment of psoriasis, who received the preparation of an dry extract of herb of anchors creeping for 3 months (1c), before the start of the treatment, the 
ratio of LH / FSH - luteinizing hormone and follicle-stimulating hormone was below normal, which indicates a certain deficit in LH production relative to FSH values. In parallel, in these cohorts, the production of prolactin was increased, which suppresses the formation of FSH and LH in the pituitary gland. Changes in the production of gonadotropic hormones are a reaction to the existing deficiency or excess of sex hormones. In the main $1 \mathrm{a}, 1 \mathrm{~b}$ and $1 \mathrm{c}$ in groups, estradiol values were within the normal range with progesterone deficiency in the luteal phase. The testosterone value in all study groups did not deviate from the standard values.

Conflicts of interest. Authors has no actual or potential conflicts of interest.

\section{References}

1. Rademaker M, Agnew K, Andrews M, Armour K, Baker C, Foley P, Frew J, Gebauer K, Gupta M, Kennedy D, Marshman G, Sullivan J. Psoriasis in those planning a family, pregnant or breast-feeding. The Australasian Psoriasis Collaboration. Australas J Dermatol. 2018 May;59(2):86-100. doi: 10.1111/ajd.12641. Epub 2017 May 23. PMID: 28543445.

2. Lockshin B, Balagula Y, Merola JF. Interleukin 17, inflammation, and cardiovascular risk in patients with psoriasis. J Am Acad Dermatol. 2018 Aug;79(2):345-352. doi: 10.1016/j.jaad.2018.02.040. Epub 2018 Mar 2. PMID: 29477740.

3. Armstrong AW, Chambers CJ, Maverakis E. Effectiveness of Online vs InPerson Care for Adults With Psoriasis: A Randomized Clinical Trial. JAMA Netw Open. 2018 Oct 5;1(6):e183062. doi: 10.1001/jamanetworkopen.2018.3062. PMID: 30646223; PMCID: PMC6324453.

4. Egeberg A, Griffiths CEM, Williams HC, Andersen YMF, Thyssen JP. Clinical characteristics, symptoms and burden of psoriasis and atopic dermatitis in adults. $\mathrm{Br} \mathbf{J}$ Dermatol. 2020 Jul;183(1):128-138. doi: 10.1111/bjd.18622. Epub 2019 Dec 4. PMID: 31630393.

5. Whitlock SM, Enos CW, Armstrong AW, Gottlieb A, Langley RG, Lebwohl M, Merola JF, Ryan C, Siegel MP, Weinberg JM, Wu JJ, Van Voorhees AS. Management of psoriasis in patients with inflammatory bowel disease: From the Medical Board of the National Psoriasis Foundation. J Am Acad Dermatol. 2018 Feb;78(2):383-394. doi: 10.1016/j.jaad.2017.06.043. PMID: 29332708. 
6. Yeung J, Gooderham MJ, Grewal P, Hong CH, Lansang P, Papp KA, Poulin Y, Turchin I, Vender R. Management of Plaque Psoriasis With Biologic Therapies in Women of Child-Bearing Potential Consensus Paper. J Cutan Med Surg. 2020 Jul/Aug;24(1_suppl):3S14S. doi: 10.1177/1203475420928376. Epub 2020 Jun 5. PMID: 32500730.

7. Kaeley GS, Eder L, Aydin SZ, Gutierrez M, Bakewell C. Enthesitis: A hallmark of psoriatic arthritis. Semin Arthritis Rheum. 2018 Aug;48(1):35-43. doi: 10.1016/j.semarthrit.2017.12.008. Epub 2018 Jan 6. PMID: 29429762.

8. Scher JU, Ogdie A, Merola JF, Ritchlin C. Preventing psoriatic arthritis: focusing on patients with psoriasis at increased risk of transition. Nat Rev Rheumatol. 2019 Mar;15(3):153-166. doi: 10.1038/s41584-019-0175-0. PMID: 30742092.

9. Merola JF, Qureshi A, Husni ME. Underdiagnosed and undertreated psoriasis: Nuances of treating psoriasis affecting the scalp, face, intertriginous areas, genitals, hands, feet, and nails. Dermatol Ther. 2018 May;31(3):e12589. doi: 10.1111/dth.12589. Epub 2018 Mar 6. PMID: 29512290; PMCID: PMC6901032.

10. Megna M, Fabbrocini G, Ruggiero A, Cinelli E. Efficacy and safety of risankizumab in psoriasis patients who failed anti-IL-17, anti-12/23 and/or anti IL-23: Preliminary data of a real-life 16-week retrospective study. Dermatol Ther. 2020 Nov;33(6):e14144. doi: 10.1111/dth.14144. Epub 2020 Sep 3. PMID: 32761740.

11. Gisondi P, Del Giglio M, Girolomoni G. Treatment Approaches to Moderate to Severe Psoriasis. Int J Mol Sci. 2017 Nov 16;18(11):2427. doi: 10.3390/ijms18112427. PMID: 29144382; PMCID: PMC5713395.

12. Papp KA, Merola JF, Gottlieb AB, Griffiths CEM, Cross N, Peterson L, Cioffi C, Blauvelt A. Dual neutralization of both interleukin 17A and interleukin 17F with bimekizumab in patients with psoriasis: Results from BE ABLE 1, a 12-week randomized, double-blinded, placebo-controlled phase 2b trial. J Am Acad Dermatol. 2018 Aug;79(2):277286.e10. doi: 10.1016/j.jaad.2018.03.037. Epub 2018 Mar 30. PMID: 29609013.

13. Lim DS, Bewley A, Oon HH. Psychological Profile of Patients with Psoriasis. Ann Acad Med Singap. 2018 Dec;47(12):516-522. PMID: 30636268.

14. Burlando M, Herzum A, Carmisciano L, Cozzani E, Parodi A. Biological therapy in genital psoriasis in women. Dermatol Ther. 2020 Jan;33(1):e13110. doi: 10.1111/dth.13110. Epub 2019 Dec 4. PMID: 31595647.

15. De Simone C, Caldarola G, Moretta G, Piscitelli L, Ricceri F, Prignano F. Moderate-to-severe psoriasis and pregnancy: impact on fertility, pregnancy outcome and 
treatment perspectives. G Ital Dermatol Venereol. 2019 Jun;154(3):305-314. doi: 10.23736/S0392-0488.18.06255-7. PMID: 31001966.

16. Kouris A, Platsidaki E, Kouskoukis C, Christodoulou C. Psychological parameters of psoriasis. Psychiatriki. 2017 Jan-Mar;28(1):54-59. doi: 10.22365/jpsych.2017.281.54. PMID: 28541239.

17. Maul JT, Navarini AA, Sommer R, Anzengruber F. Gender and age significantly determine patient needs and treatment goals in psoriasis - a lesson for practice. $\mathbf{J}$ Eur Acad Dermatol Venereol. 2019 Apr;33(4):700-708. doi: 10.1111/jdv.15324. Epub 2019 Jan 15. PMID: 30388318.

18. Martínez-Ortega JM, Nogueras P, Muñoz-Negro JE, Gutiérrez-Rojas L, González-Domenech P, Gurpegui M. Quality of life, anxiety and depressive symptoms in patients with psoriasis: A case-control study. J Psychosom Res. 2019 Sep;124:109780. doi: 10.1016/j.jpsychores.2019.109780. Epub 2019 Jul 18. PMID: 31443809. 